\title{
Expansion of human NOD/SCID-repopulating cells by stem cell factor, Flk2/Flt3 ligand, thrombopoietin, IL-6, and soluble IL-6 receptor
}

\author{
Takahiro Ueda, ${ }^{1}$ Kohichiro Tsuji, ${ }_{1}^{1}$ Hiroshi Yoshino, ${ }^{1}$ Yasuhiro Ebihara, ${ }^{1}$ \\ Hiroshi Yagasaki, ${ }^{1}$ Hiroaki Hisakawa, ${ }^{1}$ Tetsuo Mitsui, ${ }^{1}$ Atsushi Manabe, ${ }^{1}$ \\ Ryuhei Tanaka, ${ }^{1}$ Kimio Kobayashi, ${ }^{2}$ Mamoru Ito, ${ }^{2}$ Kiyoshi Yasukawa, ${ }^{3}$ \\ and Tatsutoshi Nakahata ${ }^{1}$
}

\begin{abstract}
${ }^{1}$ Department of Clinical Oncology, The Institute of Medical Science, The University of Tokyo, Tokyo 108-8639, Japan ${ }^{2}$ Central Institute for Experimental Animals, Kawasaki 216-0001, Japan

${ }^{3}$ Biotechnology Research Laboratory, Tosoh Corp., Ayase 252-1123, Japan

Address correspondence to: Kohichiro Tsuji, Department of Clinical Oncology, The Institute of Medical Science, The University of Tokyo, 4-6-1 Shirokanedai, Minato-ku, Tokyo 108-8639, Japan.

Phone: 81-3-5449-5397; Fax: 81-3-5449-5428; E-mail: tsujik@ims.u-tokyo.ac.jp.
\end{abstract}

Received for publication September 30, 1999, and accepted in revised form February 14, 2000.

\begin{abstract}
Here, we demonstrate a significant ex vivo expansion of human hematopoietic stem cells capable of repopulating in NOD/SCID mice. Using a combination of stem cell factor (SCF), Flk2/Flt3 ligand (FL), thrombopoietin (TPO), and a complex of IL-6 and soluble IL-6 receptor (IL-6/sIL-6R), we cultured cord blood CD $34^{+}$cells for 7 days and transplanted these cells into NOD/SCID mice. Bone marrow engraftment was judged successful when recipient animals contained measurable numbers of human $\mathrm{CD}^{+} 5^{+}$cells 10-12 weeks after transplantation. When cells were cultured with $\mathrm{SCF}+\mathrm{FL}+\mathrm{TPO}+\mathrm{IL}-6 /$ sIL-6R, 13 of 16 recipients were successfully engrafted, and CD $45^{+}$cells represented $11.5 \%$ of bone marrow cells in engrafted recipients. Cells cultured with a subset of these factors were less efficiently engrafted, both as measured by frequency of successful transplantations and prevalence of $\mathrm{CD}_{4} 5^{+}$cells. In animals receiving cells cultured with all 4 factors, human $\mathrm{CD}^{2} 5^{+}$cells represented various lineages, including a large number of $\mathrm{CD} 34^{+}$cells. The proportion of $\mathrm{CD} 45^{+}$cells in recipient marrow was 10 times higher in animals receiving these cultured cells than in those receiving comparable numbers of fresh $\mathrm{CD} 34^{+}$cells, and the expansion rate was estimated at 4.2 -fold by a limiting dilution method. Addition of IL-3 to the cytokine combination abrogated the repopulating ability of the expanded cells. The present study may provide a novel culture method for the expansion of human transplantable hematopoietic stem cells suitable for clinical applications.
\end{abstract}

J. Clin. Invest. 105:1013-1021 (2000).

\section{Introduction}

There has been great interest in ex vivo expansion of human long-term repopulating hematopoietic stem cells (LTR-HSCs) for a variety of developing clinical applications including HSC transplantation and gene therapy. Because the development of HSC is thought to be regulated, at least in part, by interactions of cytokine receptor signals, many investigators used various combinations of cytokines that have been shown to act on primitive hematopoietic cells to obtain the optimal culture condition for HSC expansion. In particular, stem cell factor (SCF) and Flk2/Flt3 ligand (FL) have been used as key cytokines for HSC expansion, because c-Kit and Flk2/Flt3, tyrosine kinase receptors for SCF and FL, respectively, were shown to transduce signals crucial for HSC development (1-5). Thrombopoietin (TPO), a ligand for c-Mpl, originally identified as a primary regulator for megakaryopoiesis, has also been shown to stimulate the expansion of primitive hematopoietic cells (6-8). In addition, we have demonstrated that gp130 signal activated by a complex of IL-6 and soluble IL-6 receptor (IL-6/sIL-6R) synergizes with c-Kit or Flk2/Flt3 signal to expand multipotential hematopoietic progenitor cells (HPCs) (9-11). On the other hand, previous reports on the effect of IL3 on the expansion of primitive hematopoietic cells were controversial even though they found it stimulates the expansion of relatively mature HPCs (12-16).

Clinically transplantable HSCs should prove to retain a long-term repopulating ability. Until recently, however, most of the human HSC expansion studies aimed at clinical application have used in vitro assays for $\mathrm{CD} 4^{+}$cells, colony forming cells in clonal culture, cobblestone area-forming cells (CAFCs), and long-term culture-initiating cells (LTC-ICs) to optimize the culture conditions $(7,17-20)$, but these surrogate assays have been shown not to reflect stem cell activity correctly (21-23). The recent development of assays measuring the ability to reconstitute human hematopoiesis in ontologically or genetically immunodeficient ani- 
mals enabled investigators to evaluate the stem cell activity of expanded hematopoietic cells (14-16, 24-26). We demonstrate here the significant expansion of human LTR-HSCs by a combination of SCF, FL, TPO, and IL-6/sIL-6R, using xenoplantation into NOD/SCID mice. IL-3 was shown to negatively affect the long-term repopulating ability of the cultured cells. Our culture system may pave the way for the clinical application of ex vivo expansion of human HSCs.

\section{Methods}

Mice. Experimental NOD/Shi-scid (NOD/SCID) mice, which possess a lack of mature lymphocytes, macrophage dysfunction, and an absence of circulating complements (27), were obtained from the Central Institute for Experimental Animals (Kawasaki, Japan). The mice were kept in microisolator cages on laminar flow racks in a clean experiment room. They were maintained on an irradiated, sterile diet and given autoclaved, acidified water.

Cytokines. Recombinant human IL-6 and sIL-6R were prepared as described previously (28). Recombinant human SCF was kindly provided by Amgen Inc. (Thousand Oaks, California, USA). Recombinant human TPO, IL-3, erythropoietin (EPO), and GM-CSF were generously provided by Kirin Brewery (Tokyo, Japan). Recombinant human G-CSF was kindly provided by Chugai Pharmaceutical Co. (Tokyo, Japan). Recombinant human FL was purchased from R\&D Systems (Minneapolis, Minnesota, USA). All the cytokines were pure recombinant molecules and were used at concentrations that induced an optimal response in methylcellulose culture of human bone marrow (BM) cells. These concentrations are $100 \mathrm{ng} / \mathrm{mL}$ for SCF, IL-6, and FL; $1,000 \mathrm{ng} / \mathrm{mL}$ for sIL-6R; $20 \mathrm{ng} / \mathrm{mL}$ for IL-3; $2 \mathrm{U} / \mathrm{mL}$ for EPO; and $10 \mathrm{ng} / \mathrm{mL}$ for TPO, G-CSF, and GM-CSF.

Cell preparation. Human umbilical cord blood (CB) was obtained during normal full-term deliveries after obtaining informed consent. Mononuclear cells (MNCs) were separated by Ficoll-Hypaque density gradient centrifugation after depletion of phagocytes with Silica (Immuno Biological Laboratories, Fujioka, Japan). CD $34^{+}$cells were purified from the MNCs by using Dynabeads M-450 CD34 and DETACHaBEAD CD34 (Dynal AS, Oslo, Norway). Approximately 95\% of the separated cells were found to be $\mathrm{CD} 34^{+}$cells by flow cytometric analysis.

Suspension culture. Purified CD $34^{+}$cells were incubated in suspension culture using a modification of the technique described previously (29). One milliliter of culture mixture containing $1 \times 10^{4}$ to $2 \times 10^{4} \mathrm{CD}^{+} 4^{+}$ cells, $\alpha$-medium (Flow Laboratories, Rockville, Maryland, USA), 20\% FBS (Hyclone Laboratories, Logan, Utah, USA), 1\% deionized fraction V BSA (Sigma Chemical Co., St. Louis, Missouri, USA), and various combinations of cytokines was incubated in 12-well tissue plates (Nunc A/S, Roskilde, Denmark) at $37^{\circ} \mathrm{C}$ in a humidified atmosphere flushed with $5 \% \mathrm{CO}_{2}$ in air. Serum-free suspension culture contained components identical to serum-containing culture, except $2 \%$ deionized crystallized BSA, $200 \mu \mathrm{g} / \mathrm{mL}$ of human transferrin (Sigma Chemical Co.), $160 \mu \mathrm{g} / \mathrm{mL}$ of soybean lecithin (Sigma Chemical Co.), $96 \mu \mathrm{g} / \mathrm{mL}$ of cholesterol (Nakalai Tesque Inc., Kyoto, Japan), and $10 \mu \mathrm{g} / \mathrm{mL}$ of human recombinant insulin (Sigma Chemical Co.) replaced fraction V BSA and FBS. One-milliliter aliquots of culture mixture were plated in 12-well tissue plates and incubated for 1 week at $37^{\circ} \mathrm{C}$ in a humidified atmosphere flushed with $5 \% \mathrm{CO}_{2}, 5 \% \mathrm{O}_{2}$, and $90 \% \mathrm{~N}_{2}$ in air.

Clonal culture. The freshly isolated $\mathrm{CD} 34^{+}$cells and their progenies in the suspension culture were incubated in methylcellulose culture at a concentration of $5 \times 10^{2}$ cells $/ \mathrm{mL}$ for $\mathrm{CD} 34^{+}$cells and $2 \times 10^{3}$ to $10 \times 10^{3}$ cells $/ \mathrm{mL}$ for cultured cells as reported previously (9, 11). One milliliter of mixture containing cells, $\alpha$-medium, $0.9 \%$ methylcellulose (Shinetsu Chemical Co., Tokyo, Japan), 30\% FBS, 1\% deionized fraction V BSA, $5 \times 10^{-5} \mathrm{M}$ mercaptoethanol (Eastman Organic Chemicals, Rochester, New York, USA) and a combination of cytokines including human SCF, IL-6, IL-3, G-CSF, GM-CSF, TPO, and EPO was plated in each $35-\mathrm{mm}$ standard nontissue culture dish (Nunc A/S), and incubated at $37^{\circ} \mathrm{C}$ in a humidified atmosphere flushed with $5 \% \mathrm{CO}_{2}$ in air. To evaluate human HPCs in BM cells of engrafted NOD/SCID mice, $1.5 \times 10^{5}$ cells $/ \mathrm{mL}$ of BM cells were incubated in methylcellulose culture containing $15 \%$ FBS, $1 \%$ deionized fraction V BSA, $5 \times$ $10^{-5} \mathrm{M}$ mercaptoethanol, and a combination of human SCF, IL-3, GM-CSF, and EPO. All cultures were done in triplicate and scored at day 14 of culture according to criteria reported previously (30). The abbreviations used for the colony types are as follows: $G$, granulocyte colonies; M, macrophage colonies; GM, granulocytemacrophage colonies; E, erythroid bursts; and MIX, mixed hematopoietic colonies. $\mathrm{G}$ and $\mathrm{M}$ colonies were included in GM colonies in some experiments.

Transplantation into NOD/SCID mice. Xenotransplantation of the freshly isolated $\mathrm{CD}_{3} 4^{+}$cells and their progenies in the suspension culture was performed with a modification of the method described previously (31). Briefly, cells were injected into 8- to 10week-old NOD/SCID mice irradiated with 2.4 Gy (60 Co) of total body irradiation through the tail vein. Because the natural killer cell activity of the NOD/Shiscid mice we used is detectable (27), the recipient mice were injected intraperitoneally with $400 \mu \mathrm{L}$ of PBS containing $20 \mu \mathrm{L}$ of anti-asialo GM1 antibody (Wako, Osaka, Japan) immediately before the cell transplantation. Identical treatments were performed on days 11,22 , and 33 after infusion of experimental cells. Mice were killed in a $\mathrm{CO}_{2}$ chamber 10-12 weeks after the transplantation. Femurs and tibiae were collected and aspirated with 5\% FBS-containing PBS to liberate BM cells. Cell suspensions were filtered through a sterile 40- $\mu \mathrm{m}$ cell strainer (no. 2340; Becton Dickinson Labware, Franklin Lakes, New Jersey, USA) to get rid of clumps and debris and were then processed for flow 

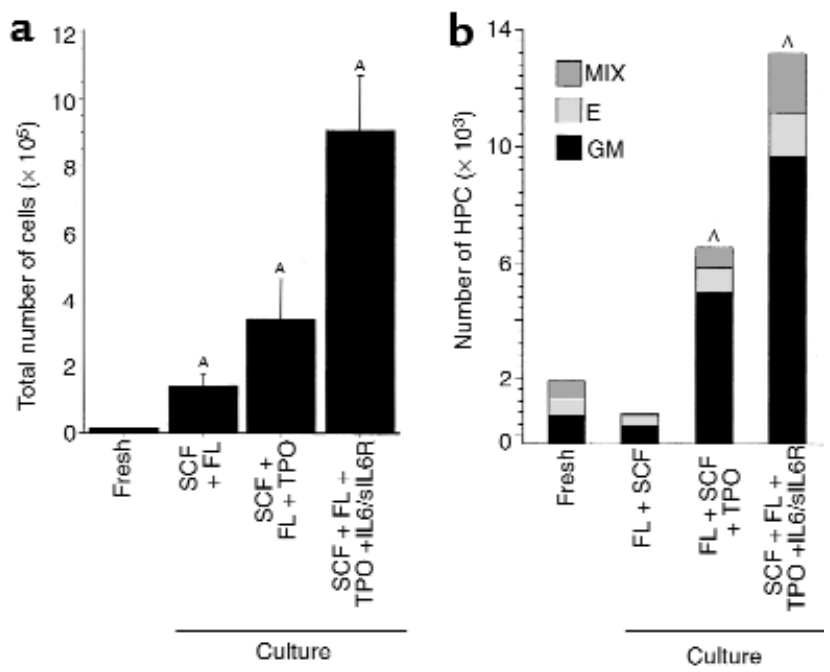

Figure 1

The expansion of total cells (a) and HPCs (b) by SCF+FL, $\mathrm{SCF}+\mathrm{FL}+\mathrm{TPO}$, and SCF+FL+TPO+IL-6/sIL-6R. A total of $2 \times 10^{4} \mathrm{CB}$ $\mathrm{CD} 34^{+}$cells from 3 samples were cultured, and the numbers of total cells and colony-forming cells in the clonal culture were analyzed at day 7 of culture. AStatistically different from data corresponding to the fresh CB CD34 ${ }^{+}$cells $(P<0.01$, Student's $t$ test $)$.

cytometric analysis and clonal culture assay. In some recipient mice, peripheral blood $(\mathrm{PB})$ collected by puncture of the tail vain was periodically analyzed until 6 months after the transplantation.

Flow cytometric analysis of transplanted NOD/SCID mice. Surface markers on human cells reconstituted in NOD/SCID mouse BM were analyzed by flow cytometry using the FACSCalibur (Becton Dickinson Immunocytometry Systems, Mountain View, California, USA) as described elsewhere (31). Briefly, $1 \times 10^{6}$ mouse BM cells suspended with $200 \mu \mathrm{L}$ of PBS containing $2 \%$ FBS were stained with antibodies at concentration of $20 \mu \mathrm{L} / \mathrm{mL}$. All antibody incubations were carried out for $30 \mathrm{~min}$ utes on ice. The presence of human hematopoietic cells was determined by detection of cells positively stained with FITC-conjugated anti-human CD45 in flow cytometric analysis. Successful engraftment by human hematopoietic cells was defined by the presence of at least $1 \%$ of human $\mathrm{CD} 45^{+}$cells in NOD/SCID mouse BM cells 10-12 weeks after the transplantation. In all recipients whose $\mathrm{BM}$ cells contained more than $1 \%$ $\mathrm{CD}^{4} 5^{+}$cells, human ALU sequences were detected in DNA extracted from the BM cells by PCR analysis as discussed later here. Specific subsets of human hematopoietic cells were quantified by gating on human CD45phycoerythrin-cyanine 5-succinimidylester-positive (PE-Cy5-positive) cells and then assessing staining with anti-human CD34-FITC, CD10-FITC, CD3-FITC, CD33-PE, CD19-PE, and CD13-PE. All antibodies were from Becton Dickinson Immunocytometry Systems (San Jose, California, ,USA) except for anti-human CD3FITC and CD45-PE-Cy5 (Immunotech, Marseille, France). For each mouse analyzed, an aliquot of cells was also stained with mouse IgG conjugated to FITC, $\mathrm{PE}$, and PE-Cy5 as isotype controls.

Limiting dilution analysis. The frequencies of human HSCs capable of repopulating in NOD/SCID mice in $\mathrm{CB} \mathrm{CD} 34^{+}$cells and their progenies in the suspension cultures were quantified by a limiting dilution analysis as described previously $(10,16,32)$. The data from several limiting dilution experiments were pooled and analyzed by applying Poisson statistics to the single-hit model. The frequency was calculated using the maximum likelihood estimator.

PCR analysis. The detection of human ALU sequences in DNA was performed by PCR analysis $(31,33)$. DNA extracted from $\mathrm{BM}$ cells of recipient mice or the colonies in clonal culture was subjected to PCR amplification using a pair of oligonucleotide primers: ALU-5, CACCTGTAATCCCAGCAGTTT-3; ALU-3, CGCGATCTCGGCTCACTGCA. Samples were denatured at $94^{\circ} \mathrm{C}$ for 4 minutes, then amplified by rounds consisting of $94^{\circ} \mathrm{C}$ for 1 minute (denaturing), $55^{\circ} \mathrm{C}$ for 45 seconds (annealing), and $72^{\circ} \mathrm{C}$ for 1 minute (extension) for 21 cycles. Products were separated on a $3.0 \%$ agarose gel, stained with ethidium bromide, and photographed.

Statistical analysis. Data are presented as the mean \pm $\mathrm{SEM}$ or mean $\pm \mathrm{SD}$. The statistical significance of the data was determined by Mann-Whitney $U$ test or Student's $t$ test. The significant level was set at 0.01 .

\section{Results}

Long-term repopulating ability of the cells cultured with single cytokines or 2-cytokine combinations. We initially examined NOD/SCID mice-repopulating ability of variable doses of fresh CB CD34 $4^{+}$cells. As shown in Table 1, none of 14 NOD/SCID mice transplanted with less than $5 \times 10^{3}$ cells revealed successful engraftment. Three $(18.8 \%)$ of 16 and $3(33.3 \%)$ of 9 recipients transplanted with $1 \times 10^{4}$ and $2 \times 10^{4}$ cells, respectively, were successfully engrafted. When $4 \times 10^{4} \mathrm{CD} 34^{+}$cells were transplanted, the high engraftment rate was obtained (66.7\%). Then, $1 \times 10^{4}$ to $2 \times 10^{4} \mathrm{CB} \mathrm{CD} 34^{+}$cells and their progenies in the culture were transplanted for analysis sensitive to the expansion of NOD/SCID-repopulating cells in the present study.

We first examined the expansion of NOD/SCIDrepopulating cells by SCF, FL, TPO, IL-6, IL-6/sIL-6R,

\section{Table 1}

Evaluation of initial cell dose of fresh $\mathrm{CD} 34^{+}$cells

Cell dose injected No. of mice reconstituted Percent of positive mice /transplanted

$1 \times 10^{3} \quad 0 / 5 \quad 0$

$5 \times 10^{3} \quad 0 / 9 \quad 0$

$1 \times 10^{4} \quad 3 / 16 \quad 18.8$

$2 \times 10^{4} \quad 3 / 9 \quad 33.3$

$\begin{array}{lll}4 \times 10^{4} & 6 / 9 & 66.7\end{array}$

NOD/SCID mice $(n=48)$ were injected with variable cell doses of fresh CB CD34+ cells and analyzed 10-12 weeks after transplantation. Successful engraftment of human hematopoietic cells was determined by detection of greater than $1 \%$ of $\mathrm{CD} 45^{+}$cells in recipient BM cells. 
Table 2

Reconstitution of NOD/SCID mice transplanted with fresh and expanded CD34+ cells

\begin{tabular}{|c|c|c|c|c|c|c|c|c|}
\hline \multirow[b]{2}{*}{ Culture conditions } & \multirow[b]{2}{*}{$\begin{array}{l}\text { No. of mice } \\
\text { reconstituted } \\
\text { /transplanted }\end{array}$} & \multicolumn{6}{|c|}{ Percent of each lineage of cells in $\mathrm{CD} 45^{+}$cells expressing } & \multirow{2}{*}{$\begin{array}{l}\text { No. of } \mathrm{CD} 34^{+} \text {cells } \\
\text { in recipients } \\
\text { engrafted }\left(\times 10^{6}\right)\end{array}$} \\
\hline & & $\begin{array}{l}\text { Percent of } \mathrm{CD} 45^{+} \\
\text {cells in } \mathrm{BM} \text { of } \\
\text { recipients }{ }^{\mathrm{B}}\end{array}$ & CD19/CD10 & CD13 & CD33 & CD3 & CD34 & \\
\hline Fresh & $6 / 25$ & $1.40 \pm 0.63$ & $85.4 \pm 3.2$ & $6.4 \pm 1.2$ & $8.7 \pm 1.0$ & 0 & $4.9 \pm 3.3$ & $0.45 \pm 0.24$ \\
\hline $\mathrm{SCF}+\mathrm{FL}$ & $7 / 16$ & $2.32 \pm 1.53$ & $72.8 \pm 1.0$ & $16.9 \pm 1.9$ & $22.0 \pm 1.0$ & 0 & $14.9 \pm 3.0$ & $1.66 \pm 2.29$ \\
\hline $\mathrm{SCF}+\mathrm{FL}+\mathrm{TPO}$ & $7 / 14$ & $6.69 \pm 3.69^{c}$ & $77.5 \pm 11.2$ & $10.7 \pm 3.9$ & $16.3 \pm 11.8$ & 0 & $16.8 \pm 6.3$ & $7.92 \pm 6.61$ \\
\hline $\begin{array}{l}\text { SCF+FL+TPO } \\
+\mid L-6 / \text { sIL-6R }\end{array}$ & $13 / 16$ & $11.47 \pm 5.87 \mathrm{C}$ & $75.1 \pm 3.2$ & $13.8 \pm 2.4$ & $13.8 \pm 2.4$ & 0 & $21.5 \pm 2.3$ & $13.72 \pm 10.65$ \\
\hline
\end{tabular}

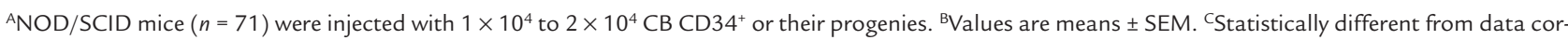
responding to mice transplanted with the fresh sample $(P<0.01$; Mann-Whitney $U$ test $)$.

or IL-3 alone. When the cells cultured from $2 \times 10^{4} \mathrm{CB}$ $\mathrm{CD} 34^{+}$cells with each of these cytokines for 1 week were injected into $3 \mathrm{NOD} / \mathrm{SCID}$ mice, no recipients revealed successful reconstitution of human hematopoietic cells, although 1 of 3 recipients transplanted with the initial $\mathrm{CB} \mathrm{CD} 34^{+}$cells did. We next evaluated the long-term repopulating ability of the cells cultured with various combinations of 2 cytokines among these cytokines. Only 2 of 3 recipients transplanted with the cells cultured with SCF+FL for 1 week were successfully engrafted, whereas all 3 transplants with the initial 2 $\times 10^{4} \mathrm{CB} \mathrm{CD} 34^{+}$cells failed. On the basis of these findings, we determined to compare the hematopoietic activities of the fresh $\mathrm{CB} \mathrm{CD} 34^{+}$cells and the cells cultured with $\mathrm{SCF}+\mathrm{FL}, \mathrm{SCF}+\mathrm{FL}+\mathrm{TPO}$, and $\mathrm{SCF}+\mathrm{FL}+$ TPO+IL-6/sIL-6R.

Reconstitution by the cells cultured from $\mathrm{CBCD} 34^{+}$cells with $S C F, F L, T P O$, and IL-6/sIL-6R in NOD/SCID mice. When $2 \times 10^{4} \mathrm{CB} \mathrm{CD} 34^{+}$cells were cultured with SCF+FL, $\mathrm{SCF}+\mathrm{FL}+\mathrm{TPO}$, and $\mathrm{SCF}+\mathrm{FL}+\mathrm{TPO}+\mathrm{IL}-6 /$ sIL-6R for 1 week, total cell numbers increased 7.2-, 17.3-, and 45.4fold, respectively $(n=3)$ as shown in Figure 1a. Morphological observation of cytospin preparations showed that there were no significant differences among the compositions of the cells cultured with the combinations, all of which contained $70-80 \%$ blastic cells. HPCs in the culture with SCF+FL did not increase, but those with $\mathrm{SCF}+\mathrm{FL}+\mathrm{TPO}$ and $\mathrm{SCF}+\mathrm{FL}+\mathrm{TPO}+\mathrm{IL}-6 / \mathrm{sIL}-6 \mathrm{R}$ increased 3.1- and 6.1-fold, respectively. The increase of multipotential HPCs was most significant in the culture with $\mathrm{SCF}+\mathrm{FL}+\mathrm{TPO}+\mathrm{IL}-$ 6/sIL-6R (3.5-fold).

We then transplanted the cells cultured from $1 \times 10^{4}$ to $2 \times 10^{4} \mathrm{CB} \mathrm{CD} 34^{+}$cells with the 3 combinations for 1 week into NOD/SCID mice and analyzed the recipient BM cells by flow cytometry 10-12 weeks after the transplantation to examine the long-term repopulating ability of the cultured cells. Figure 2 shows a representative result of flow cytometric analysis of $\mathrm{BM}$ cells in recipients engrafted with the progenies of $2 \times 10^{4} \mathrm{CB} \mathrm{CD} 34^{+}$cells obtained from the same sample. The recipient mouse transplanted with $2 \times 10^{4} \mathrm{CD}_{3} 4^{+}$cells possessed $12.0 \%$ human $\mathrm{CD}^{2} 5^{+}$ cells in BM. When NOD/SCID mice were transplanted with the cells cultured with $\mathrm{SCF}+\mathrm{FL}$ and $\mathrm{SCF}+\mathrm{FL}+\mathrm{TPO}$,
$23.6 \%$ and $39.0 \%$ of BM cells were CD45 positive, respectively. In the recipient transplanted with the cells cultured with SCF+FL+TPO+IL-6/sIL-6R, $68.5 \%$ were $\mathrm{CD} 45^{+}$cells. The expression of lineage markers on the reconstituted human $\mathrm{CD}^{2} 5^{+}$cells was analyzed (Figure 3 ). All the recipients transplanted with the cells cultured under the 3 conditions possessed $\mathrm{CD} 13^{+}$and $\mathrm{CD} 33^{+}$myeloid cells, $\mathrm{CD} 19^{+}$ and/or CD $10^{+}$B-cells, and CD $34^{+}$immature cells, but not $\mathrm{CD}^{+}{ }^{+}$-cells, in $\mathrm{BM} \mathrm{CD} 45^{+}$cells, as did the recipient engrafted with the initial CD $34^{+}$cells.

Table 2 summarizes the results of the transplantation of the cultured cells. Six (24.0\%) of 25 NOD/SCID mice transplanted with $1 \times 10^{4}$ to $2 \times 10^{4} \mathrm{CB} \mathrm{CD} 34^{+}$cells showed successful engraftment. When the cells generated from $1 \times 10^{4}$ to $2 \times 10^{4} \mathrm{CB} \mathrm{CD} 34^{+}$cells in 1 -week culture were transplanted, the rate of success increased. In the transplantation of the cells cultured with $\mathrm{SCF}+\mathrm{FL}$ and $\mathrm{SCF}+\mathrm{FL}+\mathrm{TPO}, 7$ (43.8\%) of 16 and 7 (50.0\%) of 14 recipients, respectively, were engrafted. The recipients transplanted with the cells cultured with $\mathrm{SCF}+\mathrm{FL}+\mathrm{TPO}+\mathrm{IL}-$ 6/sIL-6R showed the highest engraftment rate; 13 (81.2\%) of 16 mice showed successful engraftment. The percentages of human $\mathrm{CD}_{4} 5^{+}$cells in $\mathrm{BM}$ cells of the recipients transplanted with $1 \times 10^{4}$ to $2 \times 10^{4} \mathrm{CB} \mathrm{CD} 34^{+}$cells and the cells cultured with SCF+FL were similarly about $2 \%$, but higher in the mice transplanted with the cells cultured with SCF+FL+TPO and SCF+FL+TPO+IL-6/sIL-6R (6.7\% and $11.5 \%$, respectively).

\section{Table 3}

Formation of human hematopoietic colonies from BM cells of $\mathrm{NOD} / \mathrm{SCID}$ mice engrafted with fresh $\mathrm{CB} C D 34^{+}$cells and their progenies

No. of colonies per $1.5 \times 10^{5} \mathrm{BM}$ cells

$\begin{array}{lcccc}\text { Culture conditions } & \text { GM } & \text { E } & \text { Mix } & \text { Total } \\ \text { Fresh } & 3.3 \pm 0.6 & 0 & 0 & 3.3 \pm 0.6 \\ \text { SCF+FL } & 9.3 \pm 5.1 & 0.7 \pm 0.6 & 0 & 10.0 \pm 4.6 \\ \text { SCF+FL+TPO } & 11.3 \pm 3.2 & 0.3 \pm 0.6 & 0 & 11.7 \pm 2.9^{\mathrm{A}} \\ \text { SCF+FL+TPO } & 37.7 \pm 4.9 & 3.0 \pm 1.7 & 1.0 \pm 1.0 & 41.6 \pm 6.8^{\mathrm{A}}\end{array}$

$+\mid \mathrm{L}-6 / \mathrm{sIL}-6 \mathrm{R}$

The clonal cultures were incubated for 14 days with human SCF, IL-3, GM$\mathrm{CSF}$, and EPO. Values are means \pm SD from triplicate cultures. ${ }^{A}$ Statistically different from data corresponding to mice transplanted with the fresh sample $(P<0.01$, Student's $t$ test $)$. 

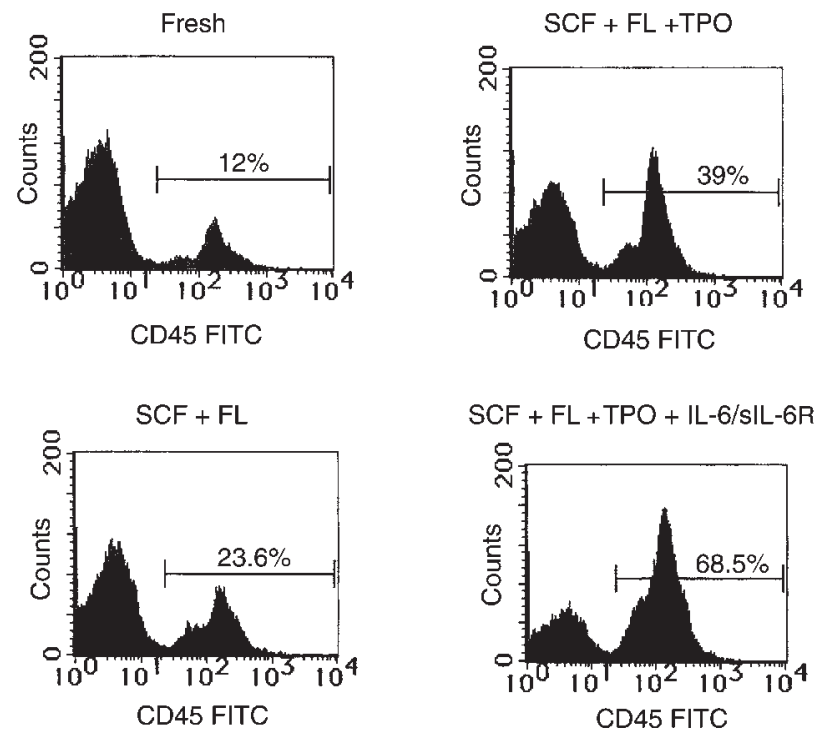

\section{Figure 2}

The repopulating ability of fresh $\mathrm{CB} C D 34^{+}$cells and the cells cultured with $S C F+F L, S C F+F L+T P O$, and SCF+FL+TPO+IL-6/sIL-6R. A total of $2 \times 10^{4} \mathrm{CB} \mathrm{CD} 34^{+}$cells and their progenies were transplanted into NOD/SCID mice, and the proportion of human CD45 cells in recipient $\mathrm{BM}$ cells was analyzed by flow cytometry 12 weeks after the transplantation.

Although B cells were the most predominantly repopulated in the recipients engrafted with the fresh CB $\mathrm{CD} 34^{+}$cells, there were no significant differences in the reconstitution of each lineage of cells among the recipients engrafted with the cultured cells. Interestingly, the proportions of human $\mathrm{CD} 34^{+}$cells in BM cells were higher in the recipients engrafted with the cultured cells than those with the initial CB cells, being only $4.9 \pm 3.3 \%$ in the latter. In particular, $\mathrm{CD} 34^{+}$cells comprised $21.5 \pm$ $2.3 \%$ of the BM cells in the recipients transplanted with the cells cultured with SCF+FL+TPO+IL-6/sIL-6R. On the basis of the data, it was estimated that 30.5 -fold of $\mathrm{CD} 4^{+}$cells were present in the recipients transplanted with SCF+FL+TPO+IL-6/sIL-6R, compared with those with fresh $\mathrm{CB} \mathrm{CD} 34^{+}$cells.

Hematopoietic activity of the cells engrafted in NOD/SCID mice. Because BM cells of the recipient mice contained a number of human $\mathrm{CD} 4^{+}$cells, the human hematopoietic activity of the reconstituted cells was examined. When $1.5 \times 10^{5} \mathrm{BM}$ cells of NOD/SCID mice untransplanted were incubated in the methycellulose clonal culture with human SCF, IL-3, GM-CSF, and $\mathrm{EPO}$, no colonies were formed, although only a small number of mouse macrophage clusters and erythroid bursts were detected. However, BM cells of the engrafted recipients shown in Figures 2 and 3 produced human hematopoietic colonies, whose numbers depended on the proportion of human $\mathrm{CD} 34^{+}$cells in each BM (Table 3). The confirmation that these colonies derived from human HPCs was obtained by the detection of human ALU sequences in DNA extracted from the colonies by PCR analysis. We ran- domly chose the colonies in the culture of BM cells of the mice engrafted with the cells cultured with SCF+FL+TPO+IL-6/sIL-6R, individually lifted the colonies from culture medium, and extracted the DNA. DNA from all of $5 \mathrm{G}$ colonies, $5 \mathrm{M}$ colonies, $5 \mathrm{GM}$ colonies, 5 E bursts and 3 MIX colonies contained human ALU sequences (data not shown).

We also monitored the presence of human $\mathrm{CD} 45^{+}$cells in $\mathrm{PB}$ of 3 recipients engrafted with the cells cultured with $\mathrm{SCF}+\mathrm{FL}+\mathrm{TPO}+\mathrm{IL}-6 / \mathrm{sIL}-6 \mathrm{R}$ until 6 months after the transplantation (Table 4). Although the proportion of $\mathrm{CD} 45^{+}$cells in $\mathrm{PB}$ was always lower than in BM, a stable number of $\mathrm{CD} 45^{+}$cells were detected for 6 months in all the engrafted recipients examined.

Expansion of buman HSCs capable of repopulating in $N O D / S C I D$ mice. The results just discussed suggest the efficient expansion of LTR-HSCs in the cultures, especially by $\mathrm{SCF}+\mathrm{FL}+\mathrm{TPO}+\mathrm{IL}-6 / \mathrm{sIL}-6 \mathrm{R}$. We then compared the proportions of human $\mathrm{CD} 45^{+}$cells in $\mathrm{BM}$ cells of NOD/SCID mice engrafted with $1 \times 10^{4}$ to $2 \times$ $10^{4}$ fresh CB CD $34^{+}$cells from 12 different samples $(1$ mouse per sample) and their progenies cultured with $\mathrm{SCF}+\mathrm{FL}+\mathrm{TPO}+\mathrm{IL}-6 /$ sIL-6R (Figure 4). Although BM cells of 8 of 12 recipients transplanted with fresh cells contained less than $1 \% \mathrm{CD} 45^{+}$cells, the reconstitution of more than $1 \% \mathrm{CD}^{4} 5^{+}$cells was obtained in BM cells of 10 mice transplanted with their progenies. In all 12 samples, the proportion of $\mathrm{CD} 45^{+}$cells in recipient $\mathrm{BM}$ cells increased by the culture with SCF+FL+TPO+IL6/sIL-6R for 1 week (mean: 10-fold; range: 1.5 - to 30.3fold; $P<0.01$ by Mann-Whitney $U$ test). Given that this finding further supported the expansion of LTR-HSCs, the expansion rate was calculated using a limiting dilution method. As shown in Figure 5, the frequency of LTR-HSCs was calculated as 1 in 39,386 in fresh CB $\mathrm{CD} 34^{+}$cells. On the other hand, the frequency of LTRHSCs in the cells cultured with $\mathrm{SCF}+\mathrm{FL}+\mathrm{TPO}+\mathrm{IL}-$ 6/sIL-6R was calculated as 1 in 9,484. Accordingly, the expansion of LTR-HSCs by SCF+FL+TPO+IL-6/sIL-6R was estimated at 4.2-fold.

Next, to examine the time required for the HSC expansion by SCF+FL+TPO+IL-6/sIL-6R, $1 \times 10^{4} \mathrm{CB}$ $\mathrm{CD} 34^{+}$cells and their progenies cultured for 4,7 , and 14 days were transplanted into NOD/SCID mice. In 4 independent experiments, no mice transplanted with the fresh $\mathrm{CB} \mathrm{CD} 34^{+}$cells and the cells cultured for 4 days possessed more than $1 \%$ of $\mathrm{CD}^{2} 5^{+}$cells in BM 12 weeks after the transplantation. However, all 4 mice transplanted with the cells cultured for 7 days were successfully engrafted. The cells cultured for 14 days did not repopulate in any of the $4 \mathrm{NOD} / \mathrm{SCID}$ mice. Thus, 7 days seemed to be an optimal duration for the HSC expansion by SCF+FL+TPO+IL-6/sIL-6R.

Reconstitution by the cells cultured with SCF, FL, TPO, and $I L-6 / s I L-6 R$ under serum-free conditions. To exclude the effects of an unknown factor(s) in FBS on the HSC expansion by SCF+FL+TPO+IL-6/sIL-6R, we transplanted $1 \times 10^{4} \mathrm{CB} \mathrm{CD} 34^{+}$cells and their progenies cultured with the cytokine combination under serum-free 

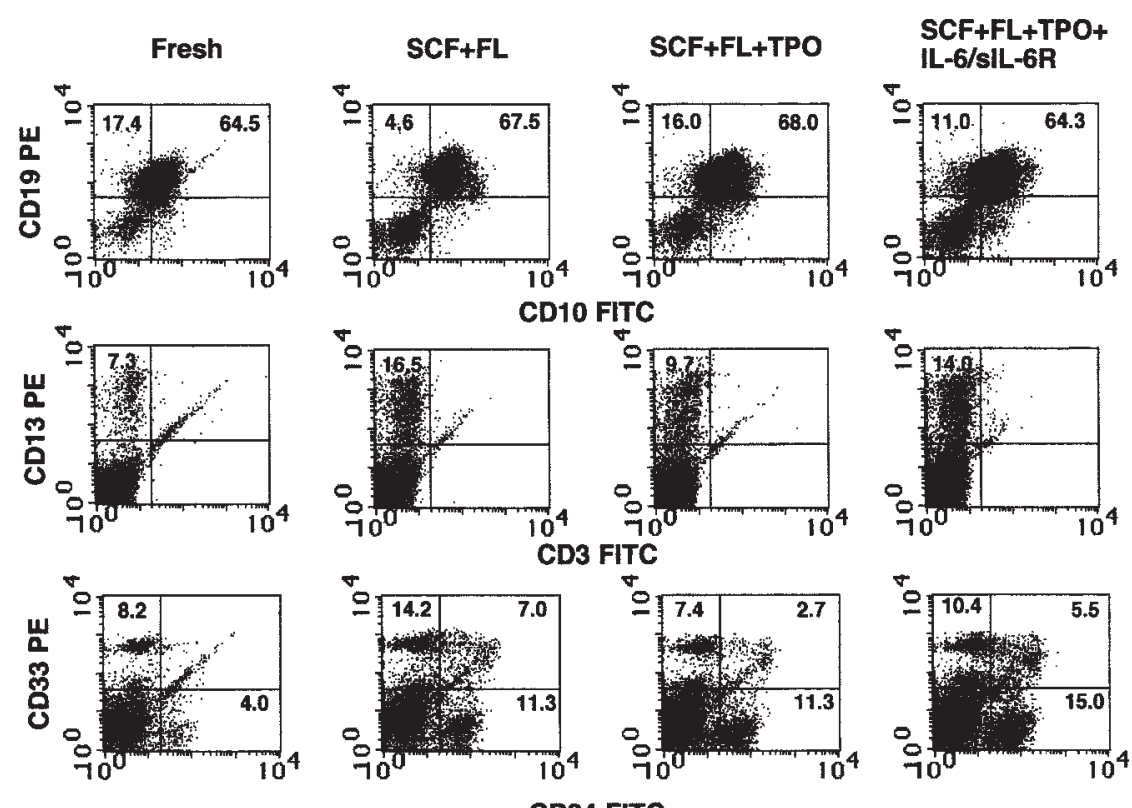

\section{Figure 3}

The expression of CD10, CD19, CD3, CD13, CD33, and CD34 on human CD45+ cells (shown in Figure 2) repopulating in BM of NOD/SCID mice engrafted with fresh $C B C D 34^{+}$cells and the cells cultured with SCF+FL, SCF+FL+TPO, and SCF+FL+TPO+IL-6/sIL-6R.

conditions into $5 \mathrm{NOD} / \mathrm{SCID}$ mice. Although no mice transplanted with the fresh $\mathrm{CB}$ CD $34^{+}$cells were successfully engrafted, 3 mice (60\%) transplanted with the cultured cells showed the reconstitution of human hematopoietic cells in BM. Flow cytometric analysis of $\mathrm{BM}$ cells of the 3 mice showed that human $\mathrm{CD} 19^{+}$cells, $\mathrm{CD} 13^{+}$cells, and $\mathrm{CD} 33^{+}$cells comprised $86.0 \pm 7.5 \%, 7.8$ $\pm 5.4 \%$, and $9.4 \pm 5.4 \%$, respectively, of the $\mathrm{CD} 45^{+}$cell population, a similar distribution to that in the recipients successfully engrafted with the cells cultured under serum-containing conditions. This result indicates that no other cytokines are needed for the HSC expansion by SCF, FL, TPO, and IL-6/sIL-6R.

Inhibitory effect of IL-3 on the expansion of human HSCs. Finally, we examined the effect of IL-3 on the expansion of human hematopoietic cells. As shown in Figure 6 , $a$ and $b$, the addition of IL-3 to the culture supplemented with SCF+FL+TPO+IL-6/sIL-6R increased the numbers of total cells and HPCs after 1 week. Then, to examine the effect of IL-3 on the expansion of LTR-HSCs, the cells in the culture of $1 \times 10^{4} \mathrm{CB}$ $\mathrm{CD} 34^{+}$cells obtained from 6 different samples with $\mathrm{SCF}+\mathrm{FL}+\mathrm{TPO}+\mathrm{IL}-6 / \mathrm{sIL}-6 \mathrm{R}$ in the presence or absence of IL-3 were transplanted into NOD/SCID mice. Although 4 of 6 recipients transplanted with the cells cultured with SCF+FL+TPO+IL-6/sIL-6R possessed more than $1 \%$ of human $\mathrm{CD} 45^{+}$cells in BM cells, no mice with the cells cultured with $\mathrm{SCF}+\mathrm{FL}+\mathrm{TPO}+\mathrm{IL}-$ $6 /$ sIL-6R+IL-3 were successfully engrafted. Figure $6 c$ summarizes the effect of the addition of IL-3 on the long-term repopulating ability of HSCs in the 4 samples that showed successful engraftment. In the transplantation of the cells cultured with IL-3, the percent- age of human $\mathrm{CD} 45^{+}$cells in BM cells was less than $0.07 \%$ in all 4 recipients $(P<0.01$; Mann-Whitney $U$ test). When $5 \times 10^{4} \mathrm{CD} 34^{+}$cells from 2 samples cultured with $\mathrm{SCF}+\mathrm{FL}+\mathrm{TPO}+\mathrm{IL}-6 / \mathrm{sIL}-6 \mathrm{R}$ in the presence or absence of IL-3 were transplanted, all the mice were successfully engrafted, but the percentages of CD $45^{+}$ cells in BM of the recipients with the cells cultured with IL-3 were lower than those without IL-3 (41.3\% and $49.5 \%$, and $63.1 \%$ and $69.3 \%$, respectively). These results indicate that IL-3 expands human mature blood cells and HPCs but has an inhibitory effect on the expansion of LTR-HSCs.

\section{Discussion}

Certain combinations of SCF, FL, TPO, and IL-6/sIL6R, such as SCF+FL, FL+TPO, SCF+IL-6/sIL-6R, FL+IL-6/sIL-6R, and SCF+FL+TPO, have been proposed for the expansion of human primitive hematopoietic cells, using in vitro assays for multilineage colony-forming cells, CAFCs, and LTC-ICs (9, 11, $17,19,34)$. The present study demonstrates that a com-

\section{Table 4}

The percentage of $\mathrm{CD} 45^{+}$cells in $\mathrm{PB}$ of the engrafted recipients

\begin{tabular}{lcccc}
\hline & \multicolumn{4}{c}{ Duration from the transplantation } \\
& 7 weeks & 12 weeks & 16 weeks & 24 weeks \\
Recipient 1 & 17.7 & 31.2 & 35.0 & 32.1 \\
Recipient 2 & 6.3 & 31.5 & 38.2 & 35.0 \\
Recipient 3 & 3.2 & 4.2 & 3.2 & 2.1
\end{tabular}

$\mathrm{NOD} / \mathrm{SCID}$ mice were injected with $1 \times 10^{4}$ to $2 \times 10^{4} \mathrm{CB} \mathrm{CD} 34^{+}$cultured with $\mathrm{SCF}+\mathrm{FL}+\mathrm{TPO}+\mathrm{IL}-6 / \mathrm{sIL}-6 \mathrm{R}$. 
bination of SCF, FL, TPO, and IL-6/sIL-6R stimulates the significant expansion of human HSCs capable of repopulating in NOD/SCID mice, and the increase in 1 week-culture was estimated at 4.2 -fold by a limiting dilution method. Because the distribution of human hematopoietic cells of each lineage reconstituted by the expanded cells in NOD/SCID mice was similar to that by the initial $\mathrm{CB} \mathrm{CD} 34^{+}$cells, it is unlikely that the cytokine combination expanded a specific population of LTR-HSCs. Furthermore, the HSC expansion by the cytokine combination was also achieved in serum-free culture, indicating that these cytokines are sufficient for the expansion.

The time-course study showed that the HSC expansion requires 1 week of culture. Considering the reports that the first cell division of CB colony-forming cells occurred within 36-60 hours in the presence of phytohemagglutinin-stimulated leukocyte-conditioned medium (35), and that the expansion of myelocytic progenitors was first observed at day 3 in the culture of $\mathrm{BM} \mathrm{CD} 34^{+}$cells with SCF and IL-3 (20), the duration required for the expansion of HSCs seems longer than that of HPCs. The dormant HSCs may take some time to begin cell cycling. Recently, it was shown that incubation with SCF and IL- 6 for 40 hours upregulated the expression of CXCR4 on $\mathrm{CD} 34^{+}$cells, resulting in increased engraftment in NOD/SCID mice (36). Therefore, one must point out the possibility that the expansion observed in the present study was caused by the upregulation of CXCR4. Given that the HSC expansion was not significant at day 4 of culture, the increased engraftment efficiency is considered not to be mediated through this mechanism.

It has not been determined whether the HSC expansion is a direct effect of SCF, FL, TPO, and IL-6/sIL-6R through the binding to their specific receptors, c-Kit, Flk2/Flt3, c-Mpl, and gp130, respectively. It was shown that a low level of c-Kit is expressed on human HSCs capable of repopulating in fetal sheep (37). c-Mpl was also shown to be expressed on human LTR-HSCs by a SCID-human bone model (38). Although the expressions of Flk2/Flt3 and gp130 on human HSCs have not been examined, Flk2/Flt3 was reported to be expressed on human CAFCs (39), and we found the expression of gp130, but not $\alpha$-chain of IL-6R, on human CD34 ${ }^{+}$ LTC-ICs (10). Taken together, these reports suggest that SCF, FL, TPO, and IL-6/sIL-6R directly act on human HSCs, even though they may affect different stages of HSCs in their expansion.

There have been only a few reports describing ex vivo expansion of human HSCs whose long term-repopulating ability was confirmed in vivo, using combinations of various cytokines $(15,16,24,25)$. However, these reports could not verify the necessity of each cytokine for the expansion. In the present study, we compared the effects of various cytokines considered to act on primitive hematopoietic cells alone or in combinations, using transplantation into NOD/SCID mice. None of SCF, FL, TPO, and IL-6/sIL-6R could solely expand or

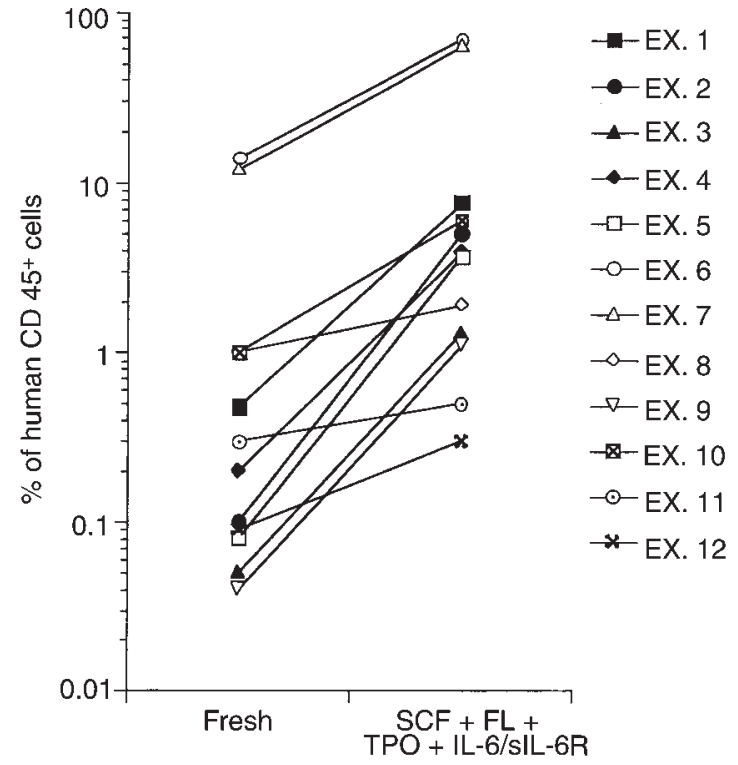

Figure 4

The comparison of the proportions of human $\mathrm{CD} 45^{+}$cells in BM cells of NOD/SCID mouse recipients transplanted with fresh CB CD34 ${ }^{+}$ cells from 12 different samples and their progenies cultured with $\mathrm{SCF}+\mathrm{FL}+\mathrm{TPO}+\mathrm{IL}-6 / \mathrm{sIL}-6 \mathrm{R}$. NOD/SCID mice were injected with $1 \times$ $10^{4}$ to $2 \times 10^{4}$ fresh $\mathrm{CB} \mathrm{CD} 34^{+}$and their progenies, and the proportions of human $\mathrm{CD} 45^{+}$cells in recipient BM cells were analyzed by flow cytometry 10-12 weeks after the transplantation. The percentages of $\mathrm{CD}_{4} 5^{+}$cells in BM cells of the recipients transplanted with the cultured cells were higher than those with fresh $C B C D 34^{+}$cells $(P<0.01$, Mann-Whitney $U$ test $)$. EX, experiment.

maintain human HSCs. Of 2-cytokine combinations, $\mathrm{SCF}+\mathrm{FL}$ maintained human HSCs without the expansion of HPCs. The addition of TPO and IL-6/sIL-6R to the combination induced significant expansion of both human HSCs and HPCs. In particular, the importance of gp130 signal in LTR-HSC expansion was recently shown using a recombinant molecule of sIL-6R fused to IL-6 (25). These observations may suggest that the synergistic action of SCF and FL supports the survival or self-renewal of human HSCs, and TPO and IL-6/sIL-6R induce the further expansion and early development of HSC in the presence of SCF and FL.

Conneally et al. and Bhatia et al. showed the expansion of human LTR-HSC by the cytokine combination including IL-3, although they did not examine the role of IL-3 in the expansion $(15,16)$. The reports on the effect of IL-3 on HSC expansion have differed for mice and humans. Culture of murine HSCs with IL-3 was detrimental to the maintenance of stem cell activity, as was shown by the repopulating ability in lethally irradiated mice $(12,13)$. By contrast, it was reported that IL-3 did not affect negatively the long-term repopulating ability of human HSCs (14). Here, we demonstrated that IL-3 exhibits an inhibitory effect on the expansion of human LTR-HSCs. Because IL-3 could stimulate the increase of mature blood cells and HPCs, IL-3 might consume human HSCs by hastening their 

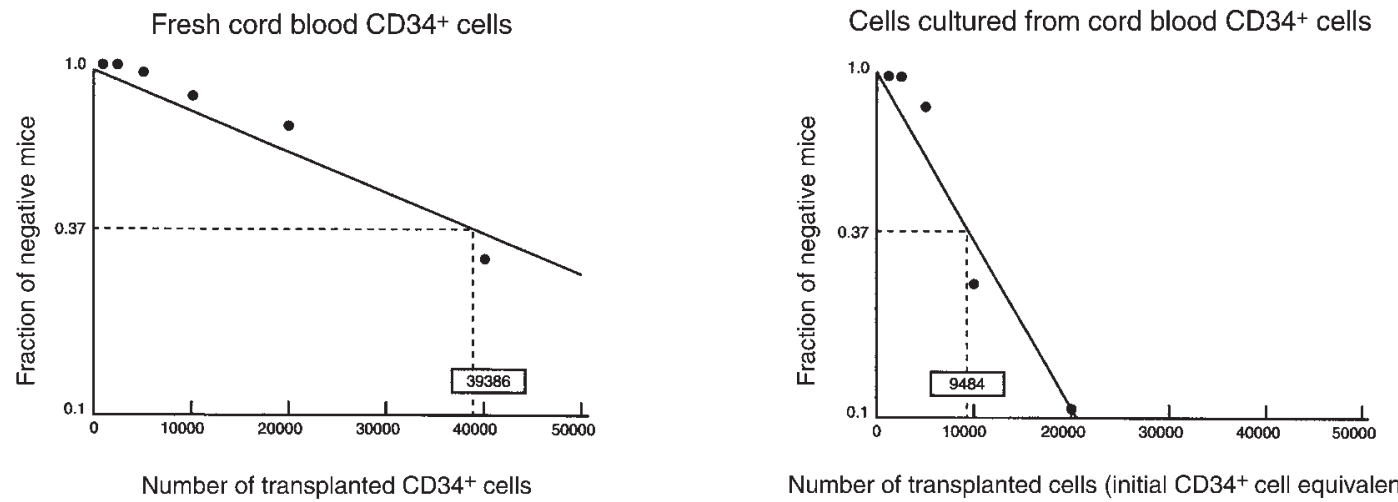

Figure 5

The frequencies of human HSCs capable of repopulating in NOD/SCID mice in fresh CB CD $34^{+}$cells $(n=52)$ and the cells cultured with SCF+FL+TPO+IL-6/sIL-6R $(n=38)$. They were estimated as 1 in 39,386 and 1 in 9,484, respectively, by a limiting dilution method.

differentiation. The discrepancy between our observation and the previous reports may be caused by the difference in the culture conditions or target cells. We also observed that in the culture of a large number of CD $34^{+}$cells, IL-3 could not completely abrogate the long-term repopulating ability of HSCs. The inoculation of a large number of HSCs may overcome the inhibitory effect of IL-3.

Many investigators have attempted to achieve efficient ex vivo expansion of human HSCs to acquire a sufficient number of transplantable HSCs in cases in which the number of useful cells obtained is limited. In particular, in CB transplantation, which is increasingly being performed as an alternative to BM transplantation, the extension of the application from children to adults is hampered by the limited quantity of $\mathrm{CB}$ harvest. In addition, the technology for HSC expansion will contribute to gene therapy, which promises to be an important approach for a number of genetic diseases and cancers. Because the use of retroviral vectors for gene transduction requires that the target cells pass through mitosis, it is necessary to stimulate HSC division while retaining the long-term repopulating ability for retroviral gene transduction into HSC. Recently, it has been reported that a combination of FL, SCF, TPO, and a fusion protein of IL- 6 and sIL-6R can induce an efficient gene transduction into human HSCs (40). In the current study, we provided a culture method using SCF, FL, TPO, and IL6/sIL-6R for the expansion of human LTR-HSCs. The increase was estimated at 4.2 -fold by a limiting dilution method, indicating that the expansion by the present culture system is practically sufficient for the application of $\mathrm{CB}$ transplantation to most adult patients. Furthermore, the expansion was achieved in serum-free culture, which in clinical application would

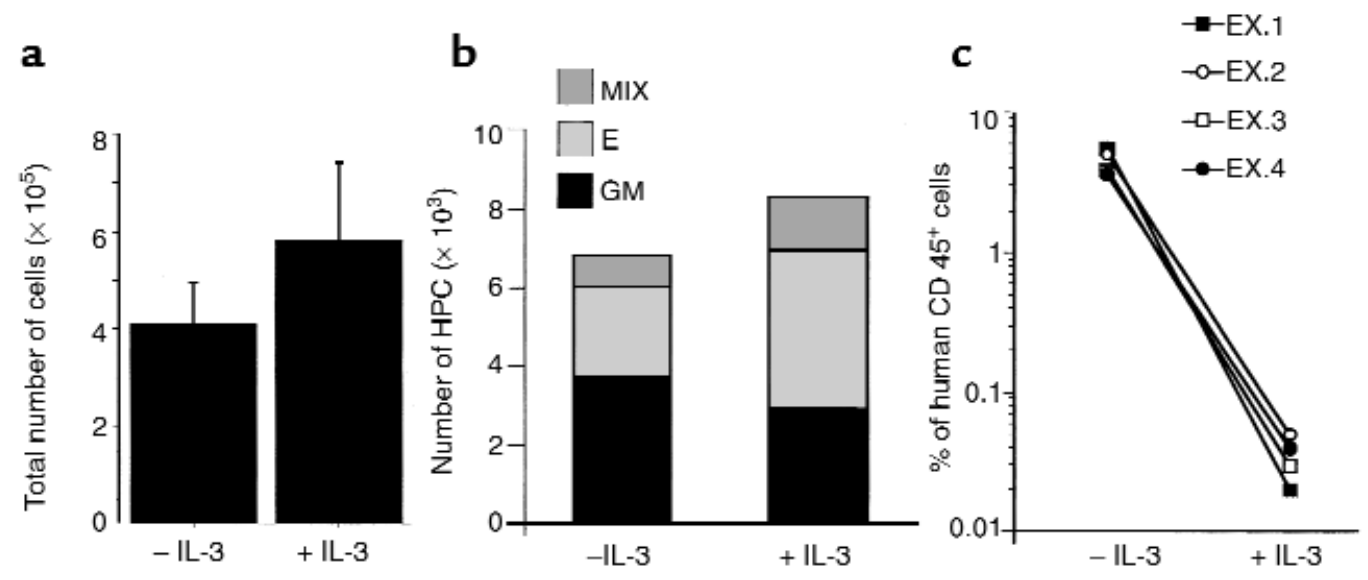

Figure 6

The effects of IL-3 on the expansion of total cells $(\mathbf{a}), \mathrm{HPCs}(\mathbf{b})$, and LTR-HSCs $(\mathbf{c})$. A total of $1 \times 10^{4}$ CB CD34 cells were cultured with $\mathrm{SCF}+\mathrm{FL}+\mathrm{TPO}+\mathrm{IL}-6 / \mathrm{sIL}-6 \mathrm{R}$ in the presence or absence of IL-3, and the numbers of total cells and colony-forming cells were analyzed at day 7 of culture. Cells cultured from 6 different samples were also transplanted into NOD/SCID mice $(n=12)$, and the proportions of human $\mathrm{CD} 45^{+}$cells in recipient BM cells were analyzed by flow cytometry 12 weeks after the transplantation. (c) The effects of the addition of IL-3 on the proportions of $\mathrm{CD} 45^{+}$cells in recipient BM cells in 4 samples whose progenies cultured with SCF+FL+TPO+IL-6/sIL-6R exhibited repopulating ability. The percentages of $\mathrm{CD} 45^{+}$cells in BM cells of the recipients transplanted with the cells cultured with IL-3 were lower than those without IL-3 $(P<0.01$, Mann-Whitney $U$ test $)$. EX, experiment. 
exclude the possibility of unknown infections. Our culture system may pave the way for the ex vivo expansion of human transplantable HSCs.

\section{Acknowledgments}

This study was supported by the Program for Promotion of Fundamental Studies in Health Sciences of the Organization for Pharmaceutical Safety and Research of Japan.

1. Williams, D.E., de Vries, P., Namen, A.E., Widmer, M.B., and Lyman, S.D 1992. The steel factor. Dev. Biol. 151:368-376

2. Rosnet, O., et al. 1993. Human FLT3/FLK2 gene: cDNA cloning and expression in hematopoietic cells. Blood. 82:1110-1119.

3. Matthews, W., Jordan, C.T., Wiegand, G.W., Pardoll, D., and Lemischka, I.R. 1991. A receptor tyrosine kinase specific to hematopoietic stem and progenitor cell-enriched populations. Cell. 65:1143-1152.

4. Small, D., et al. 1994. STK-1, the human homolog of Flk-2/Flt-3, is selectively expressed in $\mathrm{CD}_{3} 4^{+}$human bone marrow cells and is involved in the proliferation of early progenitor/stem cells. Proc. Natl. Acad. Sci. USA. 91:459-463.

5. Osawa, M., et al. 1996. In vivo self-renewal of c- $\mathrm{Kit}^{+} \mathrm{Sca}-1^{+} \mathrm{Lin}(\mathrm{low} /-)$ hemopoietic stem cells. J. Immunol. 156:3207-3214.

6. Young, J.C., et al. 1996. Thrombopoietin stimulates megakaryocytopoiesis, myelopoiesis, and expansion of $\mathrm{CD} 34^{+}$progenitor cells from single CD34 ${ }^{+} \mathrm{Thy}^{-1}{ }^{+} \mathrm{Lin}^{-}$primitive progenitor cells. Blood. 88:1619-1631.

7. Kobayashi, M., Laver, J.H., Kato, T., Miyazaki, H., and Ogawa, M. 1996. Thrombopoietin supports proliferation of human primitive hematopoietic cells in synergy with steel factor and/or interleukin-3. Blood. 88:429-436.

8. Yoshida, M., et al. 1997. Thrombopoietin alone stimulates the early proliferation and survival of human erythroid, myeloid and multipotential progenitors in serum-free culture. Br. J. Haematol. 98:254-264.

9. Sui, X., et al. 1995. gp130 and c-Kit signalings synergize for ex vivo expansion of human primitive hemopoietic progenitor cells. Proc. Natl. Acad. Sci. USA. 92:2859-2863.

10. Tajima, S., et al. 1996. Analysis of interleukin 6 receptor and gp130 expressions and proliferative capability of human CD $34^{+}$cells. J. Exp. Med. 184:1357-1364.

11. Ebihara, Y., et al. 1997. Synergistic action of Flt3 and gp130 signalings in human hematopoiesis. Blood. 90:4363-4368.

12. Yonemura, Y., Ku, H., Hirayama, F., Souza, L.M., and Ogawa, M. 1996. Interleukin 3 or interleukin 1 abrogates the reconstituting ability of hematopoietic stem cells. Proc. Natl. Acad. Sci. USA. 93:4040-4044.

13. Knobel, K.M., et al. 1994. Long-term reconstitution of mice after ex vivo expansion of bone marrow cells: differential activity of cultured bone marrow and enriched stem cell populations. Exp. Hematol. 22:1227-1235.

14. Shimizu, Y., Ogawa, M., Kobayashi, M., Almeida-Porada, G., and Zanjani, E.D. 1998. Engraftment of cultured human hematopoietic cells in sheep. Blood. 91:3688-3692.

15. Conneally, E., Cashman, J., Petzer, A., and Eaves, C. 1997. Expansion in vitro of transplantable human cord blood stem cells demonstrated using a quantitative assay of their lympho-myeloid repopulating activity in nonobese diabetic-scid/scid mice. Proc. Natl. Acad. Sci. USA. 94:9836-9841.

16. Bhatia, M., et al. 1997. Quantitative analysis reveals expansion of human hematopoietic repopulating cells after short-term ex vivo culture. J. Exp. Med. 186:619-624.

17. Petzer, A.L., Hogge, D.E., Landsdorp, P.M., Reid, D.S., and Eaves, C.J. 1996. Self-renewal of primitive human hematopoietic cells (long-termculture-initiating cells) in vitro and their expansion in defined medium. Proc. Natl. Acad. Sci. USA. 93:1470-1474.

18. Hao, Q.L., Shah, A.J., Thiemann, F.T., Smogorzewska, E.M., and Crooks, G.M. 1995. A functional comparison of CD $34^{+} \mathrm{CD}^{-} 8^{-}$cells in cord blood and bone marrow. Blood. 86:3745-3753.

19. Luens, K.M., et al. 1998. Thrombopoietin, kit ligand, and flk2/flt3 ligand together induce increased numbers of primitive hematopoietic progenitors from human CD $34^{+}$Thy $-1^{+}{ }^{2}$ in $^{-}$cells with preserved ability to engraft SCID-hu bone. Blood. 91:1206-1215

20. Bernstein, I.D., Andrews, R.G., and Zsebo, K.M. 1991. Recombinant human stem cell factor enhances the formation of colonies by CD $34^{+}$ and CD $34^{+}$lin $^{-}$cells, and the generation of colony-forming cell progeny from CD34 ${ }^{+}$lin $^{-}$cells cultured with interleukin-3, granulocyte colonystimulating factor, or granulocyte-macrophage colony-stimulating factor. Blood. 77:2316-2321.

21. Larochelle, A., et al. 1996. Identification of primitive human hematopoietic cells capable of repopulating NOD/SCID mouse bone marrow: implications for gene therapy. Nat. Med. 2:1329-1337.

22. Bhatia, M., Wang, J.C.Y., Kapp, U., Bonnet, D., and Dick, J.E. 1997. Purification of primitive human hematopoietic cells capable of repopulating immune-deficient mice. Proc. Natl. Acad. Sci. USA. 94:5320-5325.

23. Gan, O.I., Murdoch, B., Larochelle, A., and Dick, J.E. 1997. Differential maintenance of primitive human SCID-repopulating cells, clonogenic progenitors, and long-term culture-initiating cells after incubation on human bone marrow stromal cells. Blood. 90:641-650.

24. Piacibello, W., et al. 1999. Engraftment in nonobese diabetic severe combined immunodeficient mice of human $\mathrm{CD}_{3} 4^{+}$cord blood cells after ex vivo expansion: evidence for the amplification and self-renewal of repopulating stem cells. Blood. 93:3736-3749.

25. Kollet, O., et al. 1999. The soluble interleukin-6(IL-6) receptor/IL-6 fusion protein enhances in vitro maintenance and proliferation of human $\mathrm{CD} 34^{+} \mathrm{CD} 38^{-/ \text {low }}$ cells capable of repopulating severe combined immunodeficiency mice. Blood. 94:923-931.

26. van der Loo, J.C., et al. 1998. VLA-5 is expressed by mouse and human long-term repopulating hematopoietic cells and mediates adhesion to extracellular matrix protein fibronectin. J. Clin. Invest. 102:1051-1061.

27. Koyanagi, Y., et al. 1997. Primary human immunodeficiency virus type 1 viremia and central nervous system invasion in a novel hu-PBL immunodeficient mouse strain. J. Virol. 71:2417-2424.

28. Yasukawa, K., et al. 1990. Purification and characterization of soluble human IL-6 receptor expressed in CHO cells. J. Biochem. (Tokyo). 108:673-676.

29. Sui, X., et al. 1996. Erythropoietin-independent erythrocyte production: signals through gp130 and c-kit dramatically promote erythropoiesis from human CD34+ cells. J. Exp. Med. 183:837-845.

30. Nakahata, T., and Ogawa, M. 1982. Hemopoietic colony-forming cells in umbilical cord blood with extensive capability to generate mono- and multipotential hemopoietic progenitors. J. Clin. Invest. 70:1324-1328.

31. Xu, M.J., et al. 1998. Stimulation of mouse and human primitive hematopoiesis by murine embryonic aorta-gonad-mesonephros-derived stromal cell lines. Blood. 92:2032-2040.

32. Wang, J.C., Doedens, M., and Dick, J.E. 1997. Primitive human hematopoietic cells are enriched in cord blood compared with adult bone marrow or mobilized peripheral blood as measured by the quantitative in vivo SCID-repopulating cell assay. Blood. 89:3919-3924.

33. Cesano, A., et al. 1992. The severe combined immunodeficient (SCID) mouse as a model for human myeloid leukemias. Oncogene. 7:827-836.

34. Piacibello, W., et al. 1997. Extensive amplification and self-renewal of human primitive hematopoietic stem cells from cord blood. Blood. 89:2644-2653.

35. Nakahata, T., et al. 1985. Single-cell origin of human mixed hemopoietic colonies expressing various combinations of cell lineages. Blood. 65:1010-1016.

36. Peled, A., et al. 1999. Dependence of human stem cell engraftment and repopulation of NOD/SCID mice on CXCR4. Science. 283:845-848.

37. Kawashima, I., et al. 1996. CD34+ human marrow cells that express low levels of Kit protein are enriched for long-term marrow-engrafting cells. Blood. 87:4136-4142.

38. Solar, G.P., et al. 1998. Role of c-mpl in early hematopoiesis. Blood. 92:4-10.

39. Rappold, I., et al. 1997. Functional and phenotypic characterization of cord blood and bone marrow subsets expressing FLT3 (CD135) receptor tyrosine kinase. Blood. 90:111-125.

40. Hennemann, B., et al. 1999. Optimization of retroviral-mediated gene transfer to human NOD/SCID mouse repopulating cord blood cells through a systematic analysis of protocol variables. Exp. Hematol. 27:817-825 